International Journal of Engineering \& Technology, $7(2.20)(2018) 260-266$
International Journal of Engineering \& Technology
WPC
Website: www.sciencepubco.com/index.php/IJET
Research paper

\title{
A Survey on Image Fusion Requirements, Techniques, Evaluation Metrics, and Its Applications
}

\author{
M.N. Narsaiah ${ }^{1 *}$, S. Vathsal ${ }^{2}$, D. Venkat Reddy ${ }^{3}$ \\ ${ }^{1}$ Associate Professor, Dept of ECE,KG Reddy College of Engineering and Technology, Hyderabad, Telangana, India. \\ ${ }^{2} K G$ Reddy College of Engineering and Technology, Hyderabad, Telangana, India. \\ ${ }^{3}$ KG Reddy College of Engineering and Technology, Hyderabad, Telangana, India. \\ *Corresponding author E-mail:narsaiahmanthena@gmail.com
}

\begin{abstract}
Fusion refers to combining two or more distinct things, the main objective of employing fusion is to generate results that provides the most detailed, reliable and accurate information possible. The image fusion is one of the main branchof data fusion. In image fusion the images are fused at different levels of images like pixel, feature and decision level. The necessity of image fusion for high resolution on multispectral and panchromatic images or realtime images for better vision. This paper reviews the general requirements of image fusion, widely used image fusion techniques such as PCA,IHS,DWT,NSCT etc.,summarizes the Quality Assessment Metrics in terms of metric, description and its principle, finally image fusion applications in various fields such as object detection, object identification, optimization and pattern reorganization, medical imaging, etc.,
\end{abstract}

Keywords:Fusion, evaluation metric, imaging, remote sensing, spatial resolution, surveillance, intensity hue saturation.

\section{Introduction}

Image fusion is the way toward combining applicable data from at least two Images into a final resultant Image. Image fusion strategies are broadly utilized as a part of different applications, for example, remote sensing, medical imaging, military, restorative imaging, mechanical autonomy, businesses, and geo-graphical science. Image fusion is a procedure of combining more than one image to improve the information content. In order to fuse Images procedures are essential as it enhances the execution of object identification systems by incorporating numerous sources of ground based imaging system ,satellite, airborne and with other related information collections. Apart from this it supports in the process of sharpen the Images, enhance image dimensional rectifications, improves definite highlights which can't be identified input Images, substitute defect information, and also provide the data sets to make better decision. It combines the related data from at least two input Images into a final output Image whichexhibits good quality fused image and holds helpful data from input images. A panchromatic Image with high decree provides structural details of the Image due to natural and manmade objects presence in the scene, multispectral Image with low resolution provides input image color information. The multi sensor image fusion main objective is to denote the visible data from more than one image with different structural representation into a one fused image without losing information. Image fusion includes benefits like improved classification, feature enhancement, creation of stereo datasets and Image sharpening. The benefits of Multisensor Image fusion gives in wide range of operations like temporal and spatial characteristics reduced ambiguity, can carried out at different levels Image like pixel, feature and decision level.

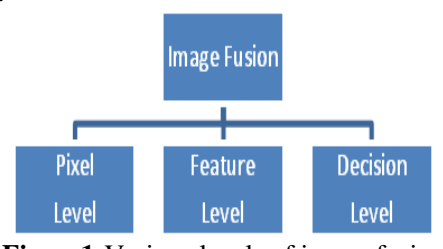

Figure1:Various levels of image fusion

Among these three levels, most broadly and very simple method used is Pixel level method. In Pixel level method, the pixels of input images are processed and retain the maximum original image information.Pixel level method provides more accurate compare to other two methods. Similarly, in Feature level process the characteristics of input images are processed for fusion and image fusion at feature level can also be used in decision level method for efficiently fusing images. The decision level method is the top level image fusion method due to decreased amount of data, it is easy to reduce and transfer the data. This method utilizes the data obtained from the feature level or pixel level fusion to formulate best possible decisions to obtain a particular objective. And also decreases uncertain information and reduce repeated information.

\section{Literature survey}

S.John Nisha Anita et al (2013) [1] clarified in their paper how to lessen color distortion because of which the information can be lost, utilizing linear pixel level fusion strategy by modifying the properties of the final resultant image.X Qing, X Shuai, T Bing, L Jiansheng(2004)[2] Image fusion is carried out using CWT instead of conventional DWT and concluded fusion system performance and improved reliability. Fusion process 
method based on CWT performs better than the fusion method based on DWT and DWPT. Firouz Abdullah Al-Wassai, N.V. Kalyankar, Ali A. Al-Zuky (2011) [3] discussed and compared various formulas of transformation matrix of HIS and concluded statistical analysis of HIS5 and YIQ are the efficient transformation based fusion methods for best representation of spectral information of multi spectral image, and the spatial details of high resolution panchromatic images. Wenkao Yang, and all (2013) [4] have proposed CS-FWT-PCA by combining classical FWT-PCA and compressed sensing(CS) and shown that the efficiency of CS-FWT-PCA is better than the conventional methods. But compressed sensing based algorithm requires more simulation time. Qiuchan Bai 1 et all (2015) [5] reviewed the literature of sensing theory and explained fundamentals of recognition method and image fusion and also compared updated compressed sensing algorithm for fusion which performs better than the commonly used and evaluated parameters like mutual information, entropy and cross entropy by analyzing wavelet transform algorithm in terms of quantitative and qualitative. Johnson Suthakar, J.Monica Esther M.E, etc.,(2014) [6] explained the aim of a image fusion process which is to produce a hybrid image with the best possible spatial data content while protecting the best spectral dataquality. Bushra N. Kayani and all (2007)[7] proposed scheme in which images are decomposed and subbands are combined with max coefficients of wavelet from two DWT coefficients. Codruta O. Ancuti, Cosmin Ancuti, Christophe De Vleeschouwer, Philippe Bekaert (2018) [8]presented an alternate method to improve underwater images and videos without any additional information than the original image, and also shown this approach is able to enhance underwater images with high accuracy and also recovers the important faded features and edges. V.V. Hnatushenko and V.V. Vasyliev (2016) [9] proposed a method to reduce the spectral distortion by combining ICA, HSV\& optimized wavelet transform, compared the new method with several existing methods using world view-2 satellite information in terms of objective and subjective aspects and concluded the fused image has better spectral quality while keeping its spatial qualities. Mirajkar Pradnya P, Ruikar Sachin D (2013) [10] explained four different methods based on wavelet transform and wavelet based image fusion and concluded hybrid architectures provides better results in all test cases and also concluded SWT based image fusion method at level 2 is outperforms than all other methods. Yosra BEN SALEM and all (2016) [11] presented normal view about fundamental models and algorithms used in the image fusion, explains different strategies which guaranties the selection of similar data and decreasing theredundancy. Aishwarya et all(2016)[12]demonstrated a novel multi-focusfusion method utilizing DWT and sparse representations and verified the propose method preserve focused regions of input images with decreased artifacts. Sourav Pramanik, and all(2013)[13]also explained algorithm for fusion based on weight calculation and regionsegmentation and verified on 120 sensor image pairs and proved that the demonstrated method achieves better results compared to the existing region based fusion algorithms and also has advantages like access to parameter setting without implementation, region classification, and better from in the view of visual perception. Yong yang(2011) [14] presented a simple DWT based algorithm for multi-focus image fusion in which the fusion method of low frequency coefficients is based on maximum sharpness algorithm whereas high frequency coefficients is based on maximum energy based selection algorithm and concluded method outperforms compared to available fusion methods. Mamta Sharma (2016) [15]Obtainedhigh PSNR and low RMSE by considering the advantages of fuzzy logic and SWT when fuzzy logic and SWT are used independently. R. Sivagam, and all(2015) [16] proposed most commonly used effective image fusion techniques, evaluation quality metrics to investigate the resultant images were reviewed and explained that every algorithms have their own merits and demerits due to fusion constraints like co-registration error, clouds and shadows present in PAN and MS images. Hai-hui Wang (2004) [17] proposed a fusion algorithm for multi-sensor images using Multiwavelet which gives less distorts spectral characteristics of the images and concluded that multiwavelet technique gives better results both visually and quantitatively. Gemma Piella(2004)[18] explained about some new objective quality measures for image fusion which don't require input image, and furthermore correlates subjective criteria with other existing performance measures. Wenzhong Shi, ChangQing Zhu, YanTian, JanetNichol(2005)[19] Addressed two issues, an analysis of image fusion methods andquality assessment for the fused images using different methods. Varsha Patil, Deepali Sale and M.A.Joshi(2013)[20] Demonstrated the combination of fusion technique such as combination of DWT and PCA(spatial domain method) improves the performance compared use of individual DWT and PCA algorithm and also mentioned visual performance based assessment methodology has its own significance.

\section{General requirements of image fusion}

After critical literature survey it is found that in order to design an image fusion system one needs consider the following requirements.

1. The fused image should retain as much as relevant information contained in the source images.

2. The fused image should remove the irrelevant features and noise to a maximum extent

3. In the Fusion process there should not be any introduction of artifacts or inconsistencies which misleads the human observer or further image processing steps.

4. In the fusion process the amount ofrelevant information should be maximum and the amount of irrelevant information, uncertainty and redundancy should be minimum in the fused image.

\section{Levels of image fusion techniques}

Depending upon the stageat which the fusion occurs, Fusion Techniques are classified as

a) Pixel Level Fusion: Image fusion at pixel level is considered as simplest technique and it is performed at lower level, image fusion at pixel level means performing integration where pixels are least processed and every pixel in resultant image is determined from pixels in the input images, like averaging

b) Feature Level Fusion: In feature level fusion, features like intensities, textures, edges are extracted from the input images depending upon similarity, selection criteria of the features fusion process takes place.

c) Decision Level Fusion: In Decision level combining the data is converged at more elevated amount of deliberation, Combines the outcomes from various algorithms to give a final decision on fused data, the input images are handled exclusively for data extraction and the acquired data is then consolidated applying choice guidelines to reinforce common interpretation. At the end of the day the result from the primary object detection and classification with the help of input images is then employed into fusion algorithm. 


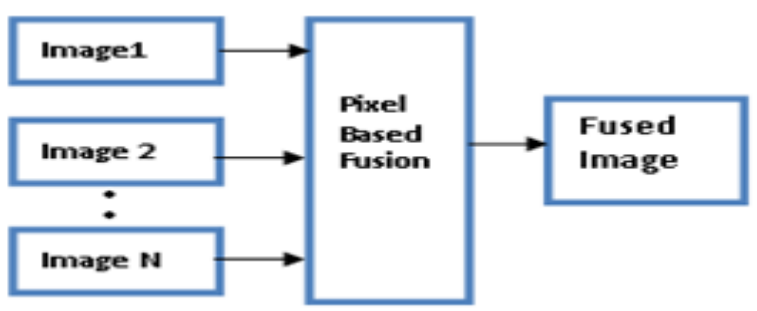

Figure 2: Pixel Based Fusion Technique.

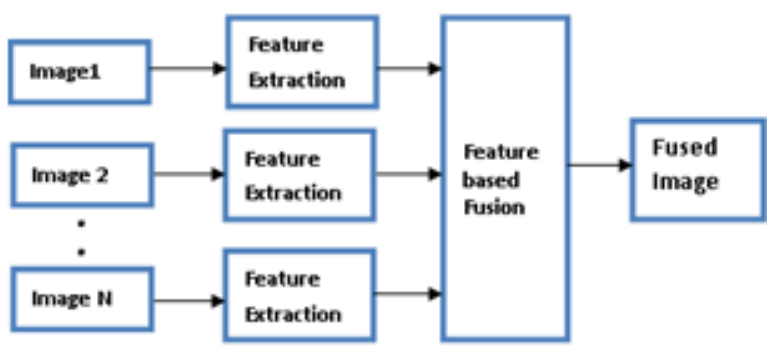

Figure 3: Feature Based Fusion Technique.

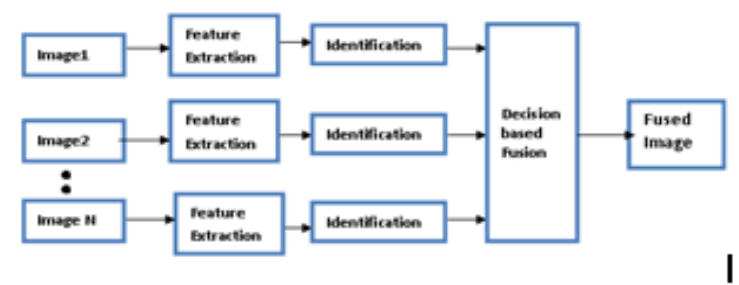

Figure 4: Decision Based Fusion Technique.

\section{Existing image fusion techniques}

- $\quad$ IHS Transform

- Principal Component Analysis (PCA)

- Brovey Transform

- Discrete Wavelet Transform

- Curvelet Transform

- Pyramid Technique

- Discrete Cosine Transform

- GA based Techniques

- Non Subsampled Contourlet Transform

\section{IHS transform}

The IHS (Intensity Hue Saturation) method is a standard methodology in image fusion, this color space is picked on the grounds that the visual subjective arrangement of human thinks about these three segments as orthogonal perceptual axis, the significant disadvantage of this system is just three bands are included, the IHS procedure is fundamentally on the RGB(Red Green Blue) real nature space in which the source images are converted from RGB(Red Green Blue) color space to the IHS space the combination is accomplished the $\operatorname{Hue}(\mathrm{H})$ and Saturation (S) band and the inverse transformation is applied to reconstruct the RGB output image.

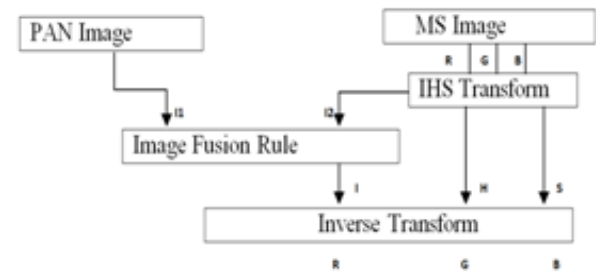

Figure 5:Process of IHS fusion

\section{Principal component analysis (PCA)}

The advantage of PCA technique over IHS technique is that any random number of bands can be utilized, which is not the case with IHS transform. PCA is a standard and popular technique among the existing image fusion techniques. Uncorrelated multi spectral images with low resolution are formed as principle components(PC 1). The PC 1 has the samedata to all bands utilized. Principle component consist more variance which gives more data about panchromatic picture. PAN component with high resolution is extended to have an identical variance from PC 1 and substitutes PC1. In order obtain the high resolution multispectral image an inverse PCA transform is applied.

\section{Brovey transform}

In this technique data generated by the sensors is combined by multiplying every MS band with high resolution PAN and divides every product by the summation of the MS band. The color normalization transform is also referred as Bovey Transform.

\section{Discrete wavelet transform (DWT) techniques}

Wavelet transform is the alternate transform to short time Fourier transform. Wavelet transform provides the required resolution in the time domain as well as in the frequency domain where as Fourier transform provides good resolution only in the frequency domain. In the conversion process of Fourier transform the given input signal is divided into two sinusoidal waves with different frequencies, where as in conversion process of wavelet transform the signal is decomposed into scaled and shifted forms of mother function or wavelet. In the process of image fusion using wavelet transform the source images are decomposed into informative and approximate coefficients using DWT at a particular level. In order to combine the coefficients a fusion rule is applied and the resulting image is obtained by taking the inverse wavelet transform.

\section{Curvlet transform}

The extended form of wavelet transform is Curvelet transform, to fuse images by using curvelet transform the following steps are involved.

- The source images are initially registered.

- Set of curvelet coefficients are generated by analyzing the each source image.

- For the fusion of curvelet coefficients the maximum frequency rule used.

- The resulting is image obtained by taking the inverse curvelet transform (ICVT).

\section{Pyramid techniques}

In the process of binocular fusion, for human visual system image pyramids are described as a model and the original 
image is represented in different levels by forming the pyramid structure. By applying pattern selective approach a composite image is formed. Initially, the pyramid decomposition on each input image is performed. Every one of individual images are combined to frame an output image, in order to get the resultant image the inverse pyramid technique is applied. At each and every level of decomposition the image fusion process is carried out and fused image can be obtained from it.

\section{Discrete cosine transform (DCT) techniques}

The DCT consider the image fusion in the frequency domain, the image fusion method which depends on average measure considered in the DCT domain. The advanced form of the direct DCT image fusion is acquired from the DCT representation of the fused imageby separating the images into blocksthen computes representation of the DCT by considering the average of all the DCT representations for corresponding blocks. Finally by taking the inverse discrete cosine transforms to get the fused image. In actuality, this image fusion technique is known as altered or "enhanced" DCT method.

\section{GA based techniques}

\section{Applications}

These are the wide applications of image fusion:

1. Remote and satellite area: Image fusion is basically used in remote and satellite area for the proper view of satellite region.

2. Medical imaging: Image fusing is used in medical imaging where disease analysis through image vision and through spatial resolution and frequency perspectives.

3. Spatial resolution: Spatial resolution of an image enhanced by the fusion of different images

4. Machine vision: Image fusion is effectively used in machine vision to visualize the two states when the image conclude its perfect for human vision

5. Robotics: used in robotics for location and recognition of object and also guides the navigation of the robot.

6. Artificial neural networks: In 3D where focal length changes according to the wavelength transformation

7. Object identification: In the process of maximize the information extracted from the images received by the satellite image fusion plays major role.

8. Classification: the accurate classification of remote sensing images is enhanced when more than one source images are introduced to the processing. One of the key task of remote sensing applications is classification

9. Facial recognition: identification and verification of a person is efficiently done by fusion thedescriptions from different images.

10. Optimization and pattern recognition: In image fusion with the help of Genetic Algorithm is used in optimization and pattern recognition problems such as function optimization, system identification, facial recognition, system identification and control

11. Object detection: Image fusion is used to detect particular class of objects from other objects in different environmental conditions to increase the situational awareness

12. Land use and land cover classification: Image fusion is used for land use and land classification since images from optical sensors and microwave offers complimentary information which helps in the process of discrimination of the deferent classes

13. Change detection: It's a process of determining differences in the state of object by observing it at
Genetic algorithm(GA) is applied to solve the problems like optimization and classification, very little research has been led on classification based image fusion by utilizing GA, , in light of the fact that in Classification based Image Fusion, the grouping issues are not that much perplexing and can be solved successfully with help of basic different classifiers. However GA can be useful in Image Fusion algorithms which require certain sort of parameter enhancements, or in other way, GA can be utilized to upgrade image Fusion process and quality by determining best value of algorithm parameters

\section{Non-sub sampled contourlet transform (NSCT)}

The technique comprises in employing the Non-Subsampled Contourlet Transform (NSCT) to divide every image to low frequency and high frequency components. Depending up on the frequency components of the two source images are merged together with the help of the directive contrast for the high frequency and stage congruency for low frequencies for the fusion. By applying inverse NSCT output fused image is obtained.

different times. In order to monitor and manage the natural resources and urban development change detection is used.

14. Ocean surveillance: tracking, detection and identification of ships, submarines.

15. Medical diagnosis: Image fusion is used to locate and identify of tumors, abnormalities and diseases.

16. Environmental monitoring: Location and identification of natural phenomena in environmental monitoring.

17. Strategic cautioning and defense: Indication of approaching vital activities can be recognized

18. Battlefield knowledge, reconnaissance and target obtaining: potential ground targets are detected and identified

\section{Conclusion}

In this literature survey, to design an image fusion system the generic requirements of image fusion should be followed. Various level of image fusion are discussed, the pixel level image fusion is simple and more accurate. The most widely used image fusion techniques like PCA, IHS, DWT, DCT, and NSCT etc., explained in detail. Quality Assessment Metrics are summarized which are considered as the key indicators of the fused image. Different domains in which image fusion can be employed are mentioned. Combining multiple fusion techniques to develop an efficient image fusion technique is the future scope of research.

\section{References}

[1] Anita SJN \& Moses CJ, "Survey on pixel level image fusion techniques", International Conference on Emerging Trends in Computing, Communication and Nanotechnology (ICE-CCN), (2013), pp.141-145.

[2] Qing X, Shuai X, Bing T \&Jiansheng L, "complex wavelets and its application to image fusion", 20th ISPRS Congress, (2004)

[3] Al-Wassai FA, Kalyankar NV \&Al-Zuky AA, "The IHS Transformations Based Image Fusion",International Journal of Advanced Research in Computer Science, Vol.2, No. 5, (2011)

[4] Yang W, Wang J \&Guo J, "A Novel Algorithm For Satellite Image Fusion Based On Compressed Sensing And PCA", Mathematical Problems in Engineering,(2013).

[5] Bai Q \&Jin C, "Image Fusion and recognition based on compressed sensing theory", International Journal on Smart Sensing and Intelligent Systems, Vol.8, No.1, (2015). 
[6] Johnson Suthakar R, Monica Esther J, Annapoorani D \&Richard Singh Samuel F, "Study of Image Fusion- Techniques, Method and Applications",IJCSMC, Vol.3, No.11, (2014), pp.469-476.

[7] Kayani BN, Mirza AM, Bangash A \&Iftikhar H, "Pixel \& feature level multiresolution image fusion based on fuzzy logic", Innovations and Advanced Techniques in Computer and Information Sciences and Engineering, (2007), pp.129-132.

[8] Ancuti CO, Ancuti C, De Vleeschouwer C \& Bekaert P, "Color balance and fusion for underwater image enhancement", IEEE Transactions on Image Processing, Vol.27, No.1,(2018), pp.379-393.

[9] Hnatushenko VV Z\&Vasyliev VV, "Remote Sensing Image Fusion Using ICA and Optimized Wavelet Transform", The International Archives of the Photogrammetry, Remote Sensing and Spatial Information Sciences,(2016).

[10] Mirajkar Pradnya P \&Ruikar Sachin D, "Wavelet based Image Fusion Techniques", International Conference on Intelligent Systems and Signal Processing (ISSP), (2013).

[11] Salem YB, Hamrouni K \& Solaiman B, "Image fusion models and techniques at pixel level", International Image Processing, Applications and Systems (IPAS), (2016), pp.1-5.

[12] Aishwarya N, Abirami S\& Amutha R, "Multifocus image fusion using Discrete Wavelet Transform and Sparse Representation", International Conference on Wireless Communications, Signal Processing and Networking (WiSPNET), (2016), pp.2377-2382.

[13] Pramanik S, Prusty S, Bhattacharjee D \& Bhunre PK, "A Region-to-pixel based Multi-sensor Image Fusion", Procedia Technology, (2013), pp.654-662.

[14] Yang Y, "A novel DWT based multi-focus image fusion method", Procedia engineering, Vol.24, (2011), pp.177-181.

[15] Sharma M, "A Review: Image Fusion Techniques and Applications",International Journal of Computer Science and Information Technologies, Vol.7, No.3, (2016), pp.1082-1085.

[16] Sivagami R, Vaithiyanathan V, Sangeetha V, Ifjaz Ahmed M, Joseph Abraham Sundar K \&Divya Lakshmi K, "Review of Image Fusion Techniques and Evaluation Metrics for Remote Sensing Applications", Indian Journal of Science and Technology, Vol.8, No.35, (2015).

[17] Wang HH, "A new multiwavelet-based approach to image fusion", Journal of Mathematical Imaging and Vision, Vol.21, No.2, (2004), pp.177-192.

[18] Piella G \& Heijmans H, "A new quality metric for image fusion", Proceedings International Conference on (Image Processing, (2003).

[19] Shi W, Zhu C, Tian Y\& Nichol J, "Wavelet-based image fusion and quality assessment", International Journal of Applied Earth Observation and Geoinformation, Vol.6,No.3-4,(2005), pp.241251.

[20] Patil V, Sale D \& Joshi MA, "Image Fusion Methods and Quality Assessment Parameters", Asian Journal of Engineering and Applied Technology, Vol.2, No.1, (2013), pp.40-46. 